\title{
Design on Urban Landscape Facilities Based on Mass Behavior
}

\author{
Yan Xia \\ Yantai Nanshan University \\ Yantai, Shandong, China 365713
}

\begin{abstract}
Outdoor activities have become people's spontaneous requirements. Pleasant and comfortable landscape facilities design will become important element in urban environmental development. Therefore, this article discusses through researching on landscape facility and mass behavior. Firstly, use observational method and case analysis method to observe behaviors and facilities in daily life and analyze typical cases of landscape facilities, and then find problems existed in the current landscape facilities design and find out the sources of the problems; then, use theoretical research method, two sources as starting points to respectively state psychology of mass behaviors, public demands and landscape facilities and find out theoretical basis of methods used to solve problems.
\end{abstract}

Keywords-mass behavior; landscape facilities; urban landscape

\section{INTRODUCTION}

Design of landscape facilities should be established on the basis of subtly analyzing today's social problems and mass behaviors. The society is always moving forward. Especially the speed of economic development of China in recent twenty years is staggering. The urbanization process is quickened. People's standards of living and ways of life have changed greatly. Urban construction is also in urgent needs of landscape facilities. Therefore, it has become requirements of this era for designers to research relationships between mass behavior and landscape facility design, and create landscape facilities that truly meet people's requirements. A lot of situations have provided opportunities as well as put forward challenges for the design of landscape facilities.

\section{CONCEPT DEFINITION OF MASS BEHAVIOR AND LANDSCAPE FACILITY}

\section{A. Mass Behavior}

Generally speaking, people's behavior refers to various activities in daily life, or people's external activities that can indicate people's thoughts, qualities and psychologies, or the process to take actions step by step in order to meet certain purposes or desire.

In mass society, mass behavior refers to collective behavior that produces special forms. It has no structure, no organization and no individual choice. When it happens, it represents the sum of many individual reactions. The mass behavior researched in this article is the gather of behaviors that happen in our daily life and in the place and seem to be optional or casual.

\section{B. Landscape Facilities}

Landscape facilities refer to man-made structures that have direct relationships with people, serve people, and have artistry or value of appreciation in certain environment. Landscape facilities are small facilities that have functions to provide places and landscape lighting for people to have a rest, decoration and display, such as garden lamp, garden seat, garden table, garden bench, machine to draw water, dustbin, sculpture, artificial hill, stone layout, fruit-rind boxes, handrail, seat, lamp-post signpost and tour guide board. The landscape facility design discussed in this article is creation activity with high quality aesthetic requirements.

\section{RESEARCH ON THE STATUS QUO OF MASS BEHAVIOR AND LANDSCAPE FACILITY}

First of all, I will give you a few cases. For example, a middle-aged man standing at the water's edge maybe like quiet places, so he chooses to see the carefree and leisurely water birds in the water. If there are wooden chairs or several rocks under the willow-tree at the water's edge for him to sit down and enjoy the scenery, maybe he will like it better. An old aunty is attracted by a water lily that is in bud, she immediately open the book and draw the outline of the beautiful scenery in front of her. Because there is no seat, she can only use one hand to hold the book and the other hand to wield the pencil. If there is a seat for her to sit down, her hands will not quiver so heavily. But on the contrary, if people stay at a place but there are no facilities, it will be inconvenient for them to have a rest. If there are large areas of hardened pavement and grasslands but without convenient facilities, when people stop at the edge of the grassland, they can only stand on the roadside; young people get together to have a party, take photos and chat, but they can only stand under the pavilion because there are no places around the pavilion for them to have a rest. It will be inevitably awkward to stand on open spaces and there are no objects to lean on.

We can observe from the above cases that the application of construction of landscape facilities in the research on the mass behavior is not perfect and appears the following 
situations: neglect the diversification of facilities, people's requirements for the sense of security, communication, personal space, and territorialism and privacy. When designing facilities, we should take people's requirements for privacy and aesthetic appreciation into full consideration. To sum up, the sources of problems existed in behaviors and facilities mainly have two reasons. One is that from the point of view of people, it neglects requirements of people's behavioral psychology; the other is that from the perspective of landscape facility, it neglects the artistry and the functions of landscape facility that enables people to appreciate the scenery and communicate.

\section{RESEARCH CONTENTS OF MASS BEHAVIOR AND LANDSCAPE DESIGN}

\section{A. Analysis on Mass Behavior}

People's basic behavior. A variety of different types of behaviors produce in the interaction between people's behavior and the environment, which is the potential of people's behavior. But people's basic behavior will not change. So we take people's basic behaviors as the starting point of the research.

The reasons for providing seats are to have conversations with others and better watch the scenery. Facilities related to seat can be divided into facilities that directly provide seats, such as bench and chair, and facilities that indirectly provide seats, such as step, edge of flower bed and retaining wall, etc. Most standing behaviors have specific functional characteristics, such as stop to wait for the traffic light to turn green, wait and see or do other things. Physical environment has little influence on this kind of very simple stay. People just stay at the places where they encounter obstacles or facades of shops or other places where they have to stop. There are two kinds of walking. On is the walking that has purpose, the other is haphazard walking. The former has the following characteristics, such as choose the best way, bypass obstacles and it is less affected by the outside world. The latter is incoherent walking movement with slow rhythm. The pedestrians are often stop-and-go and see.

Communication between people and the transmission of information are acquired through conversation. In the open air, people often chat with companions, acquaintances and strangers. The chat may be short greeting, also can be agreeable long chat. And the chat can happen at any place and time.

What will be inspected here are spontaneous activities and social activities happened in the place.

\section{B. Research on Landscape Facility}

1)Classification of landscape facility. The contents of landscape facility are extensive. There are different methods to classify landscape facility according to different conditions. This article does research on landscape facilities in the site and divides landscape facilities into three types: viewing type of landscape facilities, communication type of landscape facilities and convenient type of landscape facilities according to requirements of people's touring behaviors.

2)Viewing type of landscape facilities. Viewing type of landscape facilities directly guides people's viewing direction. The multiple layout and direction of landscape facilities can increase the viewing angle, guide people's gazing direction, improve the environmental quality of the site, increase people's aesthetic view and enhance people's aesthetic judgments. The appreciation of this kind of facilities can be achieved through combination with the surrounding environments, or the perfect manifestation of materials and modeling of facilities, or rhythm combination of many facilities. This type of landscape facilities can guide people to stop and enjoy the scenery.

3)Communication type of landscape facilities. The purpose of communication type of landscape facilities is to provide places for people to communicate, including rest facilities and recreation facilities. People often communicate with others at places that have these two types of facilities. Rest type of landscape facilities is one of the most common facilities that have the highest frequency of utilization in cities. It is an important element of urban landscape; what's more, it has strong utility function. Rest facilities can meet people's requirements to have a rest. Meanwhile, it is also one of the platforms for people to communicate. The other one is recreation facility. People can communicate with others when they play, which is the most common scene in our daily life. Adults take their children out to play beside the sandpit. Aged people play chess with many people surrounding them at the table. These recreational facilities are one of the platforms for people to enhance understanding and communication.

4)Convenient type of landscape facilities. Convenient type of landscape facilities are essential elements for integration of urban space environment. It not only provides essential devices for people's rest, exchange, activities and communication at places for outdoor activities in the city, and provides convenience for people's daily use, but also is an important part of city environment.

\section{LANDSCAPE FACILITIES DESIGN IN MASS BEHAVIOR}

\section{A. Key Points of Landscape Facilities Design}

Landscape facility exists in the place as a kind of symbol, mark and culture. Symbol type of landscape facilities occupies a very small part in the place, but facility is also a kind of symbol in the whole place and it can represent a kind of form in the place. Mark type of landscape facilities is a kind of mark in the place. It is a method and way to provide striking key points for the place. A kind of distinct mark type of landscape facilities is the sculpture. Facilities with characteristics can serve as the mark in specific environment. Culture type of landscape facilities is the symbol of culture. The cultural accumulation of historical sites can be expressed through facilities. We can use the form of symbol or the technique of abstraction to endow landscape facilities with new contents and meanings. 


\section{B. Design Procedure of Landscape Facilities}

The current design procedures firstly finish the plane layout. First of all, people see whether it can be put into the planar graph and the effect of drawing is beautiful or not, without considering that the design objects show what kind of appearance in front of visitors. They don't analyze from the perspectives of people who stand and stay, and think about how people feel or take action in it. That is to say, designers don't design by acting as a person who stays in it. So when they turn their sights to these landscape spaces, the feelings obtained by them are interrupted, fragmental and also vague. In order to improve the current design procedure of landscape facilities, we should have a clear understanding for the current design procedure of landscape facilities.

1) Determine the design objects. The determination of design objects is to confirm the types of landscape facilities that need to be introduced by the place. While the selection of facilities needs to correspond to behaviors in the place. For example, we need to provide facilities convenient for people to communicate if people have communication behaviors. The most common facilities of straight line are not convenient for people's communication. So we can consider annular facilities and facilities of broken line, etc.

2) Analyze limitations. The first is behavior in the place. Behaviors in the place mainly refer to communication behaviors and viewing behaviors. If communication behavior is the primary behavior, landscape facility design will consider how to better promote the communication. If viewing behavior is the main behavior, landscape facility design will consider how to better provide viewing. The next is the theme of the place. Theme of the place is determined according to different functions of using and must correspond to characteristics of the place.

3) Determine design elements. Good planning and design of scenic regions are the premises to guide people to appreciate the scenery. The planning and design of scenic regions involve the division of scenic regions, the set of passageways as well as landscape layout. Generally speaking, with different divisions of scenic regions, the landscape provided for people to appreciate is different, so people's feelings are different. We should base on the mass behaviors predicted in the scenic regions, consider the district planning and design of scenic regions, to make the place in scenic regions is richer and bring more viewing angles.

4) Layout of landscape facilities. The main principle of layout of landscape facilities is to promote communication and viewing. Take the facility of seat as an example, the frequency of utilization of seats that can enable people to appreciate the surrounding activities is higher than seats that are difficult for people to see others. When people choose a seat to sit down, the direction and view are also important elements. Seats that face the sun and enable people to get away from the wind and observe surrounding activities are selected by people at first. But some seats will make people uncomfortable or turn around, which cause the situation that many people will stand instead of sitting down on the existing seats in order to have a face-to-face talk.

\section{The Sizes of Landscape Facilities}

Human engineering is an important factor to determine the sizes of facilities. The most authoritative International Ergonomics Association defines it as: Human engineering is to research various factors of people such as anatomy, physiology and psychology appeared in some kind of working environment and the mutual effect between human, machine and the environment. It is the science to research the problems faced by people about how to give unified consideration to work efficiency, health, safety and comfort. In detailed design of landscape facility design, we should refer to related data in human engineering and design relevant sizes of landscape facilities. Only by doing this can landscape facilities better serve us.

\section{Materials of Landscape Facilities}

The selection of materials for landscape facilities is also an important link of landscape facility design. The use of materials or elaborate combination of different materials often brings fun for tourists. For example, the combination of stone and wood can bring us a kind of appetency that comes from the nature; the combination of glass and metal will bring us modern and light feelings.

Landscape facilities should take people who have color weakness and blind person into consideration. It is necessary to choose materials with concave-convex texture to provide touchable indicative facilities. Besides, children like to touch and obtain important information from it. It requires that the materials of facilities should be touchable and safe.

\section{E. Colors of Landscape Facilities}

Color is the design element that is easiest to move people in landscape facilities. Landscape facilities with good color processing can bring people boundless cheerfulness and pleasure. The color of each landscape facility cannot be too complicated. It should have a unified dominant color and be decorated with other colors. Thus the hue of landscape facilities can be unified and have characteristics. Therefore, the design of landscape facilities should satisfy people's use and their behavior patterns, as well as meet the requirements of people's visual aesthetic. This kind of landscape facility design is the best design.

\section{F. Image Elements of Landscape Facilities}

The image design of landscape facilities determines the artistry of facility. Its image should be the finishing touch of the place. Peculiar images can attract people's attention and also attract people to use facilities when they are appreciating. Image design is often decided by characteristics of materials. For example, we can sculpture stones and design them into different shapes, but stones have no ductility. It's nearly impossible to make them into hook face. While we can design metal materials through moulds and get different shapes.

\section{CONCLUSION}

Landscape facility design is a dynamic development process that keeps pace with the times. With the 
development of the times, mass behavior also has earthshaking changes. Designers cannot design by sticking to the old methods, but should confirm the research objectives, predict the mass behavior in the site and analyze limitations of behaviors in the place, and then determine design elements. In order to realize the interaction between landscape facilities and mass behavior, it is necessary to reasonably design the process and provide a feasible method for landscape facilities design to make landscape facilities serve us better.

\section{REFERENCES}

[1] Qian Jian, Song Lei, Design of Building Exterior Environment, Shanghai: Tongji University Press, 2001

[2] Xu Jun, Tao Kaishan. Introduction to Human Engineering, Beijing: China Textile Press, 2002.11

[3] Bai Dexuan, Beijing Institute of Architectural Design, Design of Urban Space and Environment, Beijing: China Building Industry Press, 2002, 05

[4] Ma Jianye, Research and Design on Urban Leisure Environment, China Machine Press, 2002. 06

[5] Chang Huaisheng, Environmental Psychology and Interior Design, Beijing: China Building Industry Press, 2000

[6] Wang Xun, Wang Zhuzhu, Urban Environmental Facility Design, Shanghai: Shanghai People's Fine Arts Publishing House, 2006.01 